\title{
An Evaluation of Technologies for the Pseudonymization of Medical Data
}

\author{
Thomas Neubauer and Mathias Kolb
}

\begin{abstract}
Privacy is one of the fundamental issues in health care today. Although, it is a fundamental right of every individual to demand privacy and a variety of laws were enacted that demand the protection of patients' privacy, approaches for protecting privacy often do not comply with legal requirements or basic security requirements. This paper highlights research directions currently pursued for privacy protection in e-health and evaluates common pseudonymization approaches against legal and technical criteria. Thereby, it supports decision makers in deciding on privacy systems and researchers in identifying the gaps of current approaches for privacy protection as a basis for further research.
\end{abstract}

\section{Introduction}

Privacy is not only a fundamental issues in health care today but a trade-off between the patient's demands for privacy as well as the society's need for improving efficiency and reducing costs of the health care system. Electronic health records (EHR) improve communication between health care providers and access to data and documentation, leading to better clinical and service quality. The EHR promises massive savings by digitizing diagnostic tests and images (cf. [4]). With informative and interconnected systems comes highly sensitive and personal information that is often available over the Internet and - what is more concerning - hardly protected. It is a fundamental right of every individual to demand privacy because the disclosure of sensitive data may cause serious problems. Insurance companies

\footnotetext{
Thomas Neubauer

Vienna University of Technology

e-mail: neubauer@ifs.tuwien.ac.at

Mathias Kolb

Secure Business Austria

e-mail: kolbesecurityresearch.ac.at
}

R. Lee, G. Hu, H. Miao (Eds.): Computer and Information Science. 2009, SCI 208, pp. 47-60 springerlink.com

(c) Springer-Verlag Berlin Heidelberg 2009 
or employers could use this information to deny health coverage or employment. Therefore, a variety of laws were enacted that demand the protection of privacy: In 2006 the United States Department of Health \& Human Service Health issued the Health Insurance Portability and Accountability Act (HIPAA) which demands the protection of patients data that is shared from its original source of collection (cf. [22]). In the European Union the Directive 95/46/EC [5], Article 29 Working Party [6], and Article 8 [3] of the European Convention for the Protection of Human Rights and Fundamental Freedoms demand the protection of health data. In order to protect patients' privacy when using, transferring and storing medical records, a variety of privacy enhancing techniques (cf. [7]) are proposed. However, only a few of these approaches comply (i) with the current legal requirements and (ii) basic security requirements. Researchers agree that more needs to be done to protect consumers' privacy against the onslaught of rapidly advancing technologies that track, store, and share sensitive data. Bruce Schneier states it this way: "If we ignore the problem and leave it to the "market" we'll all find that we have almost no privacy left". This development has profound implications for our society and our freedoms; it influences the way we think and live. In this discussion privacy is often not the main concern, but surveillance, and the effects it has - both positive and negative on human values, relationships, and daily practice.

This paper presents an evaluation of current privacy enhancing technologies that specifically aim at protecting medical data and, thus, can be used as a basis for EHR systems. In the scope of this paper we regard evaluation as the "systematic assessment of the operation and/or the outcomes of a program or policy, compared to a set of explicit or implicit standards, as a means of contributing to the improvement of the program or policy" (cf. [25]). We use a combination of a testing programs approach and objectives-based approach (cf. [11]). The objectives used for the evaluation are taken from the legal acts HIPAA and the EU Directive as well as from literature. This evaluation provides management decision makers such as chief privacy officers and chief security officers with a funded decision-making basis for the selection of privacy-enhancing technologies in the e-health area. As literature does not provide evaluations focusing on the comparison of PETs in e-health so far, this paper provides a major contribution to the research area of privacy.

\section{Description of Selected Pseudonymization Approaches}

Legal requirements and the explosion in privacy invasive technologies have encouraged the investment of substantial effort in the development of privacy enhancing technologies: Anonymization is the removal of the identifier from the medical data. It is realized by deleting the patient's identification data and leaving the anamnesis data for secondary use. Although this approach (and improvements ) are often used in research projects due to its simplicity, it has the major drawback that patients cannot profit from the results made in the research project. Depersonalization comprises the removal of as much information as needed to conceal the patient's identity. Encryption assures patient's privacy by encrypting the anamnesis data with 
the patient's private key. Encrypted data cannot be used for research projects (secondary use) without explicit allowance by the patient who has to decrypt the data and, thus, unconceals his identity. Pseudonymization allows an association with a patient only under specified and controlled circumstances. Pseudonymization is a technique where identification data is replaced by a specifier (pseudonym) that can only be associated with the identification data by knowing a certain secret. This chapter describes major pseudonymization approaches in detail. Thereby, we differentiate between pseudonymization approaches that store patient's data (i) encrypted and (ii) unencrypted.

\subsection{Peterson Approach}

Peterson [13] claims to provide a system for making available personal medical information records to an individual without jeopardizing privacy. The main ideas behind the approach are (i) the encryption of patient's data, (ii) the universal access to medical records by any (also unauthorized) person while (iii) the patient is responsible for granting privacy. The user registers at the provider's website, receives a unique Global Key $(G K)$ and server side key $(S S I D)$ generated by the provider and has to provide a unique Personal Encryption Key $(P E K)$ as well as a password. $G K$, $P E K$ and password are stored in the "Data Table". The user is demanded to enter a $P E K$ until he provides a unique one. After registration the user may print the GK on an ID Card (on paper).

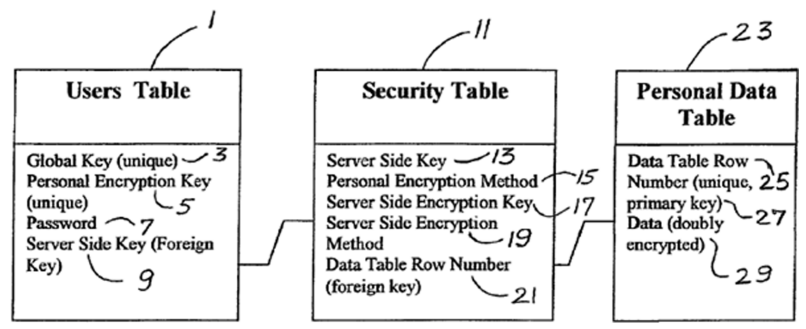

Fig. 1 Peterson Approach

This approach consists of three database tables: a "Security Table" that links the data in the "Data Table" (using attribute SSID) to the appropriate entries in the "User Table" (using attribute data table row number). Data is stored double encrypted in the database. If the user wants to retrieve data from the database, the user enters the GK or PEK which are sent to the server through a firewall and checked if the match any entry in the database. The user enters an arbitrarily key and gets immediate access to the records without authentication. The server looks up the SSID and all corresponding data table row numbers needed for retrieving the (medical) data entries from the database. The records are decrypted and delivered to the user. At this time, internal staff or malware on the health care provider's computer could 
monitor the patients' key. If a person knows the global key $G K$ or $P E K$ or both, but does not have a password, she is able to view medical data sets. To be able to add, modify or delete medical datasets, the person has to provide an additional password. Peterson arguments, that this access levels protect the privacy of a patient, because the data does not contain any identifying information. So, for an attacker, it would be uninteresting to receive anonymous data. The approach of Peterson [13] provides a fall-back mechanism, if the patient has lost or used her $G K$. Therefore the patient has to login to the system with her $P E K$ and password. Afterwards she requests a new $G K$, which could be printed on a new card. The new $G K$ assures, that her medical data is protected against unauthorized access using the old key.

\subsection{Pseudonymization of Information for Privacy in e-Health}

Pseudonymization of Information for Privacy in e-Health (PIPE) [15, 16, 12] introduces a new architecture that provides the following contributions compared to other methodologies: (i) authorization of health care providers or relatives to access defined medical data on encryption level, (ii) secure fall-back mechanism, in case the security token is lost or worn out, (iii) data storage without the possibility of data profiling, and (iv) secondary use without establishing a link between the data and the owner. The client is a service, which provides an interface to legacy applications, manages requests to local smart card readers and creates a secure connection to the server. The server, also called Logic $(\mathrm{L})$, handles requests from clients to the storage. The data in the storage is divided into two parts, the personal data and the pseudonymized medical data. As shown in figure 2, the link between personal data and pseudonymized medical data is protected through a hull-architecture. The hullarchitecture contains a minimum of three security-layers: the authentication layer (outer hull), the user permission layer (inner hull) and the concealed data layer. To reach the next hull, there are one or more secrets, for example, symmetric or asymmetric keys or hidden relations, in every hull-layer. PIPE defines users with different roles comprising patient $A$, relative $B$, health care provider $C$ or operator $O$. The patient is the owner of her data and has full control of her datasets. She is able to view her medical data, add and revoke health care providers and she may define relatives, who have the same rights as herself. Health care providers can be authorized to see and create subsets of anamnesis data by the patient. The operators are the administrators of the system.

- The authentication layer contains an asymmetric key pair, for example the patient outer public key $K_{A}$ and outer private key $K_{A}^{-1}$. These keys are stored on a smart card and are protected with a pin code. The outer private key is used to decrypt the keys of the permission hull-layer.

- The permission layer contains an asymmetric key pair and a symmetric key, for example the patient inner public key $\widehat{K}_{A}$, inner private key $\widehat{K}_{A}^{-1}$ and symmetric key $\bar{K}_{A}$. The symmetric key is encrypted with the inner private key and is used to en-/decrypt pseudonyms in the concealed data layer. If a patient associates 


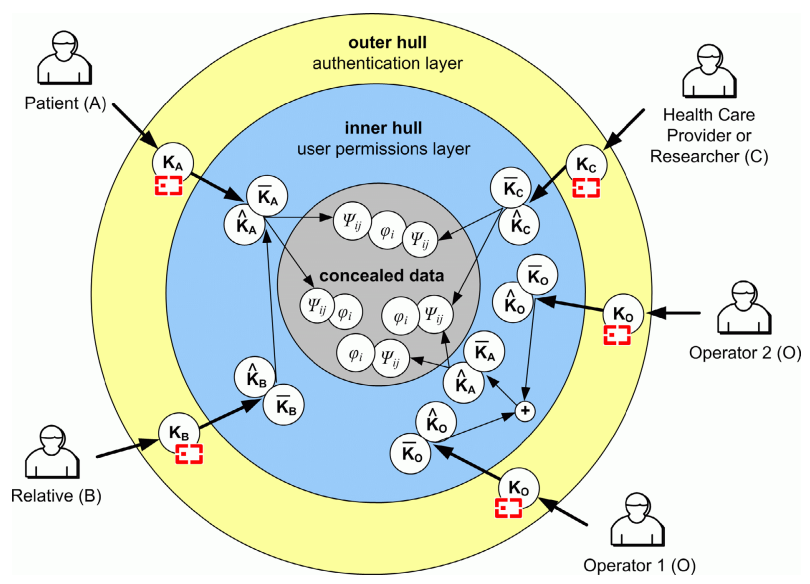

Fig. 2 PIPE: Layered model representing the authorization mechanism

a relative, her inner private key $\widehat{K}_{A}^{-1}$ will be encrypted with the relative's inner public key $\widehat{K}_{B}$. So, the relative is able to decrypt the patient's symmetric key $\bar{K}_{A}$ with her inner private key $\widehat{K}_{B}^{-1}$, until the patient's inner private key $\widehat{K}_{A}^{-1}$ is changed.

- The concealed data layer contains hidden relations, which are called pseudonyms. Each medical data set is associated with one or more pseudonyms $\psi_{i_{j}}$. As the patient is the owner of her medical data and the person with security clearance, she owns the so called root-pseudonym $\psi_{i_{0}}$. These pseudonyms are calculated with an algorithm, which on the user's symmetric key. Only instances, who are able to decrypt one of these pseudonyms $\psi_{i j}$, can rebuild the link between the patient and her medical data.

To find the pseudonyms to rebuild the link to the medical data, the authors introduced keywords. Keywords are selected on creation time of the medical data or when another user is authorized. They are encrypted with the symmetric key of the root user and the authorized users. After the keywords are stored in the database, the user can select any of these keywords to find the pseudonym. As all data would be lost if a patient loses her smart card or the smart card is worn-out, PIPE implements a fall-back mechanism to replace the smart card. Therefore, "operators" $O$ have been introduced, who share the patient's inner private key $\widehat{K}_{A}^{-1}$. Therefore the patient's inner private key $\widehat{K}_{A}^{-1}$ is divided into shared secrets by the use of Shamir's threshold scheme [18]. This scheme allows sharing keys between several operators. The key is shared with $N_{A}\left(N_{A} \subset N\right)$ randomly assigned operators and to recover the key, $N_{k}\left(N_{k} \subseteq N_{A}\right)$ operators are needed. The operators have no knowledge which keys they hold. 


\subsection{Electronic Health Card (eGK)}

The electronic health card [8, 2] is an approach of the Fraunhofer Institute supported by the Federal Ministry of Health in Germany. EGK is designed as a serviceoriented architecture (SOA) with some restrictions. One of these restrictions is, that the health card can only be accessed locally on the client side. Another restriction is, that services should use remote procedure calls for communication due to performance and availability issues. Therefore, the system architecture is divided into five layers.

1. The presentation layer defines interfaces to communicate with the user.

2. The business logic layer combines different services, which are processed automatically.

3. The service layer provides special functional uncoupled services.

4. The application layer primarily realizes the user right and data management.

5. The infrastructure layer contains all physical hardware and software management, for example, data storage, system management, virtual private networks, etc.

With this layered architecture, the system provides several service applications such as emergency data, electronic prescription, electronic medical report or a electronic health record system. The system includes a ticketing concept to realize some uncoupled action in combination with security mechanisms, to comply with the privacy policy: All data, which will be stored in the virtual filesystem is encrypted with a one-time symmetric key, called session key. This session key is encrypted with the public key of the patient. To decrypt the data, the patient has to decrypt the session key with his private key and finally the data is decrypted with this session key. A user is authenticated by using a challenge-response approach. Therefore the system generates a random number that is encrypted with the user's public key. Only the user is allowed to decrypt this random number with his private key, which is stored on her health card and can send it back to the eGK system. Furthermore, the ticketing concept manages the access rights to the system. A file or directory in this virtual filesystem has a default ticket-toolkit and any amount of private tickettoolkits, called t-node). The user defines a private ticket-toolkit for every other user in the system. This private ticket-toolkit can have stronger or looser access policies than the default ticket-toolkit. The ticket-toolkit contains a ticket-building tool, a ticket-verifier, the access policy list and a encrypted link to the directory or file. Every user holds a root directory in the virtual filesystem. Any directory contains unencrypted links to the ticket-toolkits of their child nodes. This technique enables the system to perform a fast selection of sub nodes (select $*$ from t-nodes where parentID = directoryID). To be able to find the root node of a specific user, the query service maps a unique identifier and returns a ticket-toolkit containing an encrypted link to the root node. If there is no private ticket-toolkit available for the user, who performed the request, the system returns a default ticket-toolkit, which is based on a challenge. If the user is able to solve this challenge, she will get the access rights, which have been defined in the default access policy. Both, the hybrid encryption 


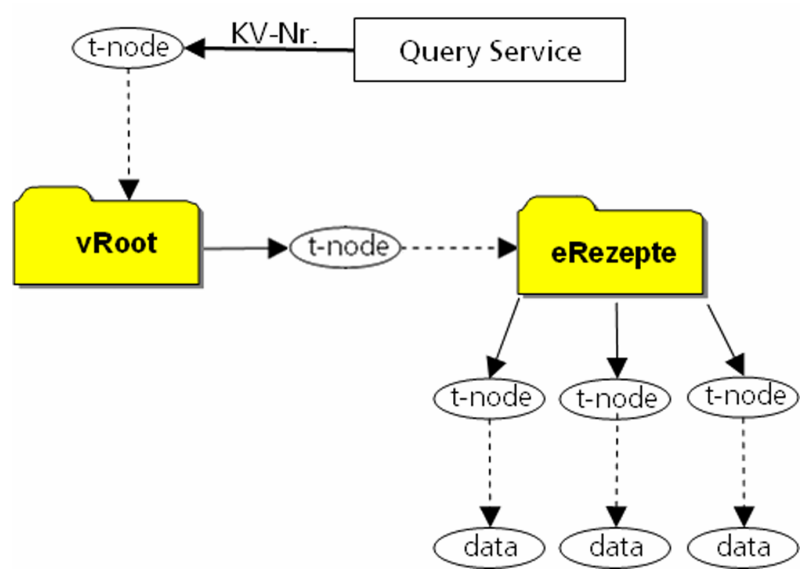

Fig. 3 eGK: Virtual filesystem [8]

and the challenge response technique are based on the asymmetric key pair, which is stored on the patients' health card. Neither the operating company nor any public administration organization could recover the data, if the patient lost the smart card or the card is worn out. To overcome this problem, the eGK architecture optionally allows to store a second private ticket-toolkit for every entry. This private tickettoolkit uses an asymmetric key pair, which is stored on an emergency card. The architecture does not specify this emergency card, but recommends to use the card of a family member or a notary. In case the card has been lost, the patient requests a new health card. Therefore, the emergency card is used to decrypt the session keys of the second ticket-toolkit and finally the session keys are encrypted with the keys of the new health card. After this process, the system does not accept the old health and emergency cards anymore.

\subsection{Thielscher Approach}

Thielscher [21] proposed a electronic health record system, which uses decentralized keys stored on smart cards. The medical data is split into identification data and the anamnesis data and stored in two different databases. The key stored on the patient's smart card is used to link the patient identity to her datasets. Therefore, this key generates a unique data identification code (DIC), which is also stored in the database. Such a DIC does not contain any information to identify an individual. Data identification codes are shared between the patient and health care providers to authorize them to access the medical data set. For more security the authorization is limited to a certain time period. After this period any access attempt is invalid. The keys to calculate the data identification code (DIC) are stored on smart cards. In case these smart cards are lost, a fall-back mechanism is provided by Thielscher. 


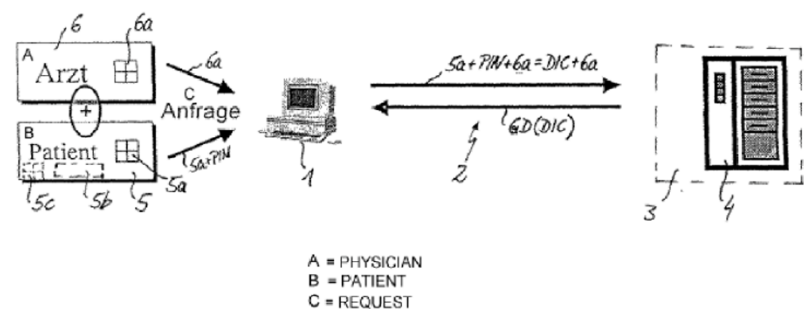

Fig. 4 Thielscher: Architecture [21]

Every pseudonym hold by a patient is stored in a list, which is stored at an off-line computer. In case the smart card is lost or destroyed, this list could be used to re-link the data to the patient.

\subsection{Approach of Slamanig and Stingl}

The approach of Slamanig and Stingl [20, 19] stores the data in a centralized database and uses smart cards for authentication. The system keeps the pseudonyms of a user secret. Each pseudonym realizes a sub-identity of the user and is encrypted with a public key. In case the user wants to view the datasets of one of his subidentities, she has to login into the system with her general pin code and she has to enter the pin code of the sub-identity to activate the private key on the smart card. Furthermore a public pseudonym of each user is available, which is used for authorization purposes. The system is divided into two repositories, the user repository and document repository. The link between these repositories is done by holding a 5-tuple dataset $\left(U_{S}, U_{R}, U_{C}, U_{P}, D_{i}\right)$, which contains the sender $U_{S}$, the receiver $U_{R}$, the creator $U_{C}$, the concerning user $U_{P}$ (e.g., the patient) and the document $D_{i}$. To ensure, that on creation time no linkage between the concerned user is possible, all elements in the tuple, are encrypted with the public key of the receiver, except of the receiver element $U_{R}$. Until the receiver has not logged into the system, the receiver element $U_{R}$ will be the public pseudonym. On the next login of the receiver, the system will replace the receiver element $U_{R}$ with a secret pseudonym of the user and re-encrypts the other elements of the tuple. As shown in figure 5 this tuple dataset can also be used for exchanging documents between users. There are six possible variations for exchanging the message:

1. $\left({ }_{-},-, U_{C}, U_{P}, D_{i}\right)$ : Creator and concerning user are known

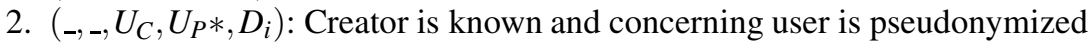

3. $\left({ }_{-},{ }_{-}, U_{C},{ }_{-}, D_{i}\right)$ : Creator is known and concerning user is anonymized

4. $\left(,,-,,-, U_{P}, D_{i}\right)$ : Concerning user is known

5. $\left(-,{ }_{-}, U_{P}, D_{i}\right)$ : Concerning user is pseudonymized

6. $\left(-,,,-,, D_{i}\right)$ : fully anonymized 


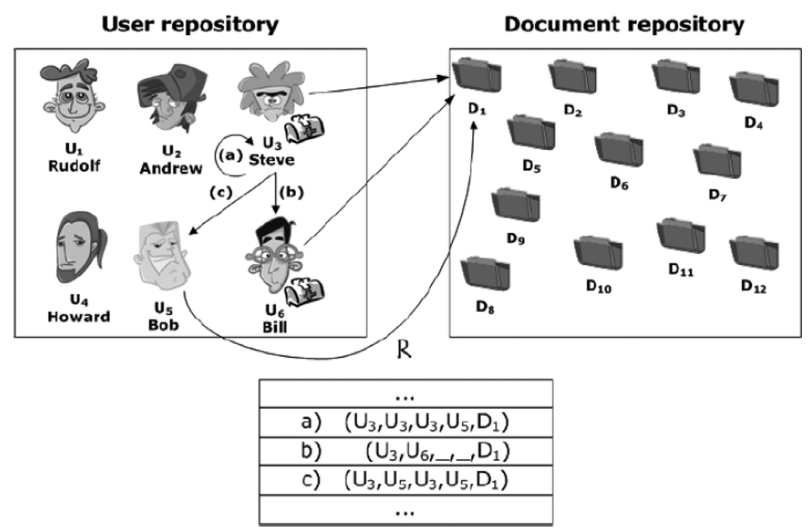

Fig. 5 Slamanig and Stingl: Repositories and Shares [19]

As fall-back mechanism, the authors mention, that a distributed key backup to $N$ users using a $(t, N)$-threshold secret sharing scheme could be implemented.

\subsection{Pommerening Approaches}

Pommerening [14] proposes different approaches for secondary use of medical data. The first approach is based on data from overlapping sources for one-time secondary use. To connect the data a unique identifier (PID) is introduced. A pseudonymization service encrypts the PID with a hash algorithm and the medical data is encrypted with the public key of the secondary user (cf. Figure 6). The secondary user can decrypt the medical data and merge the data of a person, but cannot identify it. The second approach is also based on one-time secondary use, but with the possibility to re-identify the patient. Therefore, Pommerening extends the first approach with a PID service, which stores a reference list containing the patient's identity and the associated PIDs. In case the patient should be notified, the pseudonymization service decrypts the pseudonym and sends the request to the PID service, which allows to notify the data source owner. The third approach fits the need of a research network with many secondary users and it also supports long-term observation of patients. The research results can be send to the patient or her responsible health care

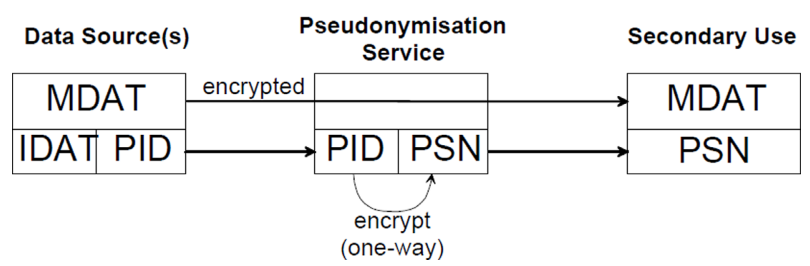

Fig. 6 Pommerening: Data Flow for One-Time Secondary Use [14] 


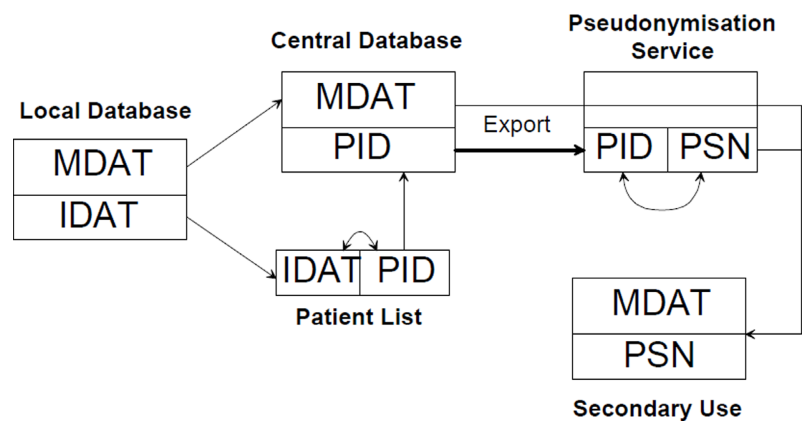

Fig. 7 Pommerening: Data Flow for multiple Secondary Uses [14]

provider. Therefore a physician export his local database to the central researcher database (cf. Figure 7). The identification data is replaced with a PID using the PID service. For each secondary use the data is exported through the pseudonymization service. The PID is encrypted by the pseudonymization service with a project specific key to ensure that different projects get different pseudonyms.

\section{Evaluation}

Pseudonymization approaches (e.g., used for securing electronic health record systems) have to adhere certain requirements to accord with privacy laws in the European Union and United States. The following set of requirements has been extracted from legal acts (cf. [5, 9, 10, 24, 23]).

- User authentication: The system has to provide adequate mechanisms for user authentication. This could be done, for example with smart cards or finger print.

- Data ownership: The owner of the medical data has to be the patient. The patient should be able to define who is authorized to access and create her medical records.

- Limited access: The system must ensure that medical data is only provided to authenticated and authorized persons.

- Protection against unauthorized and authorized access: The medical records of an individual have to be protected against unauthorized access. This includes system administrators who should not be able to access these medical records, for example, through compromising the database.

- Notice about uses of patients data: The patient should be informed about any access to her medical records.

- Access and copy own data: The system has to provide mechanisms to access and copy the patients own data.

Additionally, pseudonymization approaches have to implement certain technical requirements in order to provide an appropriate level of security (cf. [16, 1]). 
- Fallback mechanism: The system should provide mechanisms to backup and restore the security token used for pseudonymization. Therefore, the system has to guarantee, that the security token could only be restored under the compliance of a four-eye-principle.

- Unobservability: means, that pseudonymized medical data can not be observed and linked to a specific individual in the system.

- Secondary use: The system should provide a mechanism to (i) export pseu-donymi-zed data for secondary use and (ii) notify the owner of the exported data, if new medicaments or treatment methods are available.

- Emergency access: In case of an emergency, the rescue service or emergency physician should have access to an emergency dataset, in which important information is saved. For example, blood group, informations about medication, allergic reactions to specific medicaments, etc.

- Insider abuse: Medical personal may abuse their access rights for own purposes. For example they want to know how their family members or celebrities are being treated. Insider do not only abuse their privileges for own purposes. They may release informations to outsiders for spite, revenge or profit [17].

- Physical compromise of the database: The system should grant that an attacker who gets physical access to the systems database cannot identify the owners of the stored data records.

- Modification of the database: If an attacker breaks into the system, the system must detect modifications and inform the system administrator about this attack.

Table 1 applies the legal and technical criteria defined above to the selected pseudo-nymi-zation approaches. Characteristics that are accurate with the law or fully implemented are denoted with $x$, whereas characteristics that are not accurate with the law or not implemented are denoted with - and $o$ indicates properties that are partially implemented.

Table 1 Evaluation of pseudonymization approaches

\begin{tabular}{|c|c|c|c|c|c|c|c|c|}
\hline Legal Requirements & $D P$ & $I I P A A$ & $P I P E$ & $\overline{e G K}$ & $P o$ & $P e$ & $T$ & \\
\hline User authentication & $\mathrm{X}$ & $\mathrm{X}$ & $\mathrm{x}$ & $\mathrm{x}$ & - & $\overline{\mathrm{o}}$ & $\bar{x}$ & \\
\hline Data ownership & $\mathrm{x}$ & $\mathrm{x}$ & $\mathrm{x}$ & $\mathrm{x}$ & - & - & $x$ & \\
\hline Limited access & $\mathrm{x}$ & $\mathrm{x}$ & $\mathrm{x}$ & $\mathrm{x}$ & $\mathrm{o}$ & - & $x$ & \\
\hline Protection against unauthorized and authorized access & $\mathrm{x}$ & $\mathrm{x}$ & $\mathrm{x}$ & $\mathrm{x}$ & $\mathrm{o}$ & - & c & \\
\hline Notice about uses of patients data & $\mathrm{x}$ & $\mathrm{x}$ & $\mathrm{x}$ & $\mathrm{x}$ & - & - & - & 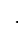 \\
\hline Access and copy own data & $\mathrm{x}$ & $\mathrm{x}$ & $\mathrm{x}$ & $\mathrm{x}$ & $\mathrm{o}$ & $\mathrm{x}$ & $x$ & 2 \\
\hline Technical Requirements & $D P$ & $\overline{I I P A A}$ & $P I P E$ & $\overline{e G K}$ & $\overline{P o}$ & & $\bar{T}$ & \\
\hline Fallback mechanism & - & - & $\mathrm{x}$ & $\mathrm{x}$ & - & $\mathrm{o}$ & $\bar{x}$ & \\
\hline Unobservability & $\mathrm{x}$ & $\mathrm{x}$ & $\mathrm{x}$ & $\mathrm{x}$ & $\mathrm{x}$ & - & $X$ & \\
\hline Secondary use & - & $\mathrm{x}$ & $\mathrm{x}$ & o & $\mathrm{x}$ & - & - & \\
\hline Emergency access & - & - & $\mathrm{x}$ & $\mathrm{x}$ & - & $\mathrm{x}$ & - & \\
\hline Insider abuse & - & - & $\mathrm{x}$ & $\mathrm{x}$ & $\mathrm{x}$ & $\mathrm{x}$ & $x$ & \\
\hline Database modifications & - & - & $\mathrm{x}$ & $\mathrm{x}$ & $\mathrm{X}$ & $x$ & $X$ & \\
\hline
\end{tabular}


The approaches of Pommerening and Peterson only pseudonymize data on export. The approaches of Pommerening have the drawback that the generated pseudonyms from the PID service are stored in a reference patient list, to be able to re-build the link to the patient. To enhance the security, this list will be stored at a third party institution, but this measure does not prevent an abuse of the list through an insider of the third party institution. An attacker could bribe an insider of the third party institution to get access to the patient list or the identifying data of some pseudonyms. The Peterson approach has some major security issues. As all keys needed for decrypting the medical data are stored in the database, an attacker getting access to the database can decrypt all information and the attacker may change data stored in the database unnoticedly, as the password and the keys are stored in the database. The $P E K$ is selected by the user but must be unique in the system. This behavior does not only open a security leak because the user trying to chose a key is informed about the keys that already existing in the system. An attacker could use the keys reported as existing for immediate access to the medical data associated with this key. Moreover, this behavior is impractical and inefficient in practice as the user might have to select dozens of keys before he enters a valid one. Although the data is doubly encrypted an attacker getting access to the database gets access to all data stored on the server because the keys needed for decrypting the data are (i) also stored in the same database and (ii) the relation between the tables (thus between the identification data and the medical data) are stored in clear text.

Thielscher's approach comes with the shortcoming, that a centralized pseudonym is centrally stored in the patient mapping list for recovery purposes. To prevent attacks to this list, Thielscher keeps this list off-line, but this mechanism cannot prevent insider abuse or social engineering attacks. Furthermore, it does not provide protection if the attacker gets physical access to the computer. Another drawback of the system is the emergency call center, that . This call center can abuse their access privileges to get access to medical data of any patient. The drawback of the approach of Slamanig and Stingl is that an attacker may authorize other users, send faked medical documents or disclose medical data. This attack is possible, because the authors use a weak mechanism for authorization and disclosure. For example, the requirements to send a faked medical document are, (i) access to the database, (ii) the public pseudonym $U_{P}$ of the user, which the attacker wants to harm, (iii) any public pseudonym to fake the sender $U_{S}$ and creator $U_{C}$, (iv) the public pseudonym and the public key $K_{R}$ of the receiver $U_{R}$, for example the employer, and (v) a harmful document $D_{i}$. After the attacker has all the required information, she inserts a new tuple into the authorization table. After the next login of the receiver, the system replaces the public pseudonym of the user with a private pseudonym of the receiver. In contrast, PIPE and eGK store the data pseudonymized in the database. Attackers who get access to the database or system administrators cannot link the data to individuals. Both approaches provide a high level of security because even if the attacker breaks into the database, she would not be able to link and read the stored data. The only way to link the data to an individual is to fake an official photo ID in order to get a new smart card that allows to access the system. Another method to link data to an individual is by doing a data mining or data profiling attack. This 
attack could be done by identified keywords, which are not encrypted in the eGK approach or by identifiable words in the anamnesis data in the PIPE approach.

\section{Conclusions}

Health care requires the sharing of patient related data in order to provide efficient patients' treatment. As highly sensitive and personal information is stored and shared within interconnected systems, there is increasing political, legal and social pressure to guarantee patients' privacy. Although, legislation demands the protection of patients' privacy, most approaches that lay claim to protect patient's privacy fail in fulfilling legal and technical requirements. This paper gave an overview of research directions that are currently pursued for privacy protection in e-health and identified pseudonymization as the most promising approach. By evaluating common pseudonymization approaches against legal and technical criteria taken from legal acts and literature, this paper answered the question which approaches fulfill the current legal requirements regarding the protection of medical data. From the six candidates that were evaluated, only two can be seriously considered for use in practice. The result show that more contemporary approaches fulfill more of the legal requirements of the European Union and the United States. Whereas the eGK approach encrypts patients' data, PIPE leaves the decision of encrypting patients' data up to the user. Therefore, PIPE turns out to be the more appropriate option if secondary use is demanded. The results of the evaluation can support decision makers (such as chief security officers) especially in health care in their decision process when it comes to the selection of a system for protecting patients' data according to legal requirements posed by HIPAA or the EU Directives. Furthermore, the results may assist researchers in identifying the gaps of current approaches for privacy protection as a basis for further research.

Acknowledgements. This work was supported by grants of the Austrian Government's FITIT Research Initiative on Trust in IT Systems under the contract 816158 and was performed at the Research Center Secure Business Austria funded by the Federal Ministry of Economics and Labor of the Republic of Austria (BMWA) and the City of Vienna.

\section{References}

1. Barrows, R.C., Clayton, P.D.: Privacy, confidentiality, and electronic medical records. Journal of the American Medical Informatics Association 13, 139-148 (1996)

2. Caumanns, J.: Der Patient bleibt Herr seiner Daten. Informatik-Spektrum, pp. 321-331 (2006)

3. Council of Europe: European Convention on Human Rights. Martinus Nijhoff Publishers (1987)

4. Ernst, F.R., Grizzle, A.J.: Drug-related morbidity and mortality: Updating the cost-ofillness model. Tech. rep., University of Arizona (2001) 
5. European Union: Directive 95/46/EC of the European Parliament and of the council of 24 October 1995 on the protection of individuals with regard to the processing of personal data and on the free movement of such data. Official Journal of the European Communities L 281, 31-50 (1995)

6. European Union, Article 29 Working Party: Working document on the processing of personal data relating to health in electronic health records (EHR) (February 2007)

7. Fischer-Hübner, S.: IT-Security and Privacy: Design and Use of Privacy-Enhancing Security. Springer, Heidelberg (2001)

8. Fraunhofer Institut: Spezifikation der Lösungsarchitektur zur Umsetzung der Anwendungen der elektronischen Gesundheitskarte (2005)

9. Hinde, S.: Privacy legislation: A comparison of the US and european approaches. Computers and Security 22(5), 378-387 (2003)

10. Hornung, G., Götz, C.F.J., Goldschmidt, A.J.W.: Die küenftige TelematikRahmenarchitektur im Gesundheitswesen. Wirtschaftsinformatik 47, 171-179 (2005)

11. House, E.R.: Assumptions underlying evaluation models. Educational Researcher 7(3), 4-12 (1978)

12. Neubauer, T., Riedl, B.: Improving patients privacy with pseudonymization. In: Proceedings of the International Congress of the European Federation for Medical Informatics (2008)

13. Peterson, R.L.: Encryption system for allowing immediate universal access to medical records while maintaining complete patient control over privacy. US Patent Application Publication, No.: US 2003/0074564 A1 (2003)

14. Pommerening, K., Reng, M.: Secondary use of the Electronic Health Record via pseudonymisation. In: Medical And Care Compunetics 1, pp. 441-446. IOS Press, Amsterdam (2004)

15. Riedl, B., Neubauer, T., Boehm, O.: Patent: Datenverarbeitungssystem zur Verarbeitung von Objektdaten. Austrian-Patent, No. A 503291 B1, 2007 (2006)

16. Riedl, B., Neubauer, T., Goluch, G., Boehm, O., Reinauer, G., Krumboeck, A.: A secure architecture for the pseudonymization of medical data. In: Proceedings of the Second International Conference on Availability, Reliability and Security, pp. 318-324 (2007)

17. Rindfleisch, T.C.: Privacy, information technology, and health care. Commun. ACM 40(8), 92-100 (1997)

18. Shamir, A.: How to share a secret. Commun. ACM 22(11), 612-613 (1979)

19. Slamanig, D., Stingl, C.: Privacy aspects of e-health. In: Proceedings of the Third International Conference on Availability, Reliability and Security, pp. 1226-1233 (2008)

20. Stingl, C., Slamanig, D.: Berechtigungskonzept für ein e-health-portal. In: Schreier, G., Hayn, D., Ammenwerth, E. (eds.) eHealth 2007 - Medical Informatics meets eHealth, vol. 227, pp. 135-140. Österreichische Computer Gesellschaft (2007)

21. Thielscher, C., Gottfried, M., Umbreit, S., Boegner, F., Haack, J., Schroeders, N.: Patent: Data processing system for patient data. Int. Patent, WO 03/034294 A2 (2005)

22. United States Department of Health \& Human Service: HIPAA administrative simplification: Enforcement; final rule. Federal Register / Rules and Regulations 71(32) (2006)

23. U.S. Congress: Health Insurance Portability and Accountability Act of 1996. In: 104th Congress (1996)

24. U.S. Department of Health \& Human Services Office for Civil Rights: Summary of the HIPAA Privacy Rule (2003)

25. Weiss, C.H.: Evaluation: Methods for studying programs and policies, 2nd edn. PrenticeHall, Englewood Cliffs (1998) 